\title{
Role of spiral volumetric computed tomographic scanning in the assessment of patients with clinical suspicion of pulmonary embolism and an abnormal ventilation/perfusion lung scan
}

Astrid B van Rossum, Frank E E Treurniet, Gerard J Kieft, Sierd J Smith, Relinde Schepers-Bok

\begin{abstract}
Background - A study was carried out to evaluate the potential place of spiral volumetric computed tomography (SVCT) in the diagnostic strategy for pulmonary embolism.

Methods - In a prospective study 249 patients with clinical suspicion of pulmonary embolism were evaluated with various imaging techniques. In all patients a ventilation/perfusion (V/Q்) scan was performed. Seventy seven patients with an abnormal $\dot{V} / \dot{Q}$ scan underwent SVCT. Pulmonary angiography was then performed in all 42 patients with a non-diagnostic $\dot{V} / \dot{Q}$ scan and in three patients with a high probability $\dot{V} / \dot{Q}$ scan without emboli on the SVCT scan. Patients with an abnormal perfusion scan also underwent ultrasonography of the legs for the detection of deep vein thrombosis.
\end{abstract}

Results - One hundred and seventy two patients $(69 \%)$ had a normal $\dot{V} / \dot{Q}$ scan. Forty two patients (17\%) had a non-diagnostic $\dot{V} / Q$ scan, and in five of these patients pulmonary emboli were found both by SVCT and pulmonary angiography. In one patient, although SVCT showed no emboli, the angiogram was positive for pulmonary embolism. In one of the 42 patients the SVCT scan showed an embolus which was not confirmed by pulmonary angiography. The other 35 patients showed no sign of emboli. Thirty five patients $(14 \%)$ had a high probability $\dot{V} / \dot{Q}$ scan, and in 32 patients emboli were seen on SVCT images. Two patients had both a negative SVCT scan and a negative pulmonary angiogram. In one who had an inconclusive SVCT scan pulmonary angiography was positive. The sensitivity for pulmonary embolism was $95 \%$ and the specificity $97 \%$; the positive and negative predicted values of SVCT were $97 \%$ and 97\%, respectively.

Conclusions - SVCT is a relatively noninvasive test for pulmonary embolism which is both sensitive and specific and which may serve as an alternative to ventilation scintigraphy and possibly to pul- monary angiography in the diagnostic strategy for pulmonary embolism.

(Thorax 1996;51:23-28)

Keywords: pulmonary embolism, pulmonary arteries, computed tomography, lung scintigraphy.

Pulmonary embolism is a major complication of venous thrombosis with an incidence in the West of 2-3 per 1000 inhabitants and a mortality rate, if untreated, of $30 \% .{ }^{1}$ Adequate anticoagulant therapy reduces the mortality rate to $8 \%,{ }^{1}$ so a diagnostic test for pulmonary embolism has to be sensitive and specific because treatment has a high complication rate. ${ }^{23}$ Symptoms and signs of pulmonary embolism are non-specific and laboratory tests do not provide an accurate diagnosis, so diagnosis relies heavily on imaging techniques. Chest radiographic and perfusion lung scan abnormalities are non-specific, although a normal perfusion lung scan essentially rules out pulmonary embolism. ${ }^{45}$ If the perfusion lung scan is abnormal, combination with a ventilation lung scan raises the specificity. A high probability $\dot{\mathrm{V}} / \mathrm{Q}$ scan gives an $86-92 \%$ chance of pulmonary embolism being present and warrants anticoagulant therapy. ${ }^{6-9}$ Unfortunately, up to $60 \%$ of the patients suspected of pulmonary embolism have an equivocal so-called nondiagnostic $\dot{V} / \dot{Q} \operatorname{scan}^{6-81015}$ and in these patients it is necessary to visualise the pulmonary arteries to rule out the presence of emboli. Pulmonary angiography is the reference standard for detecting clots, but is invasive. The challenge is to find a less invasive test to identify the true positive and negative patients amongst those with a non-diagnostic V/Q scan.

Computed tomographic (CT) scanning with intravenous contrast medium is relatively noninvasive and the development of electron beam CT scanning and spiral volumetric CT (SVCT) scanning makes it possible to depict contrast enhanced pulmonary arteries down to segmental and subsegmental levels using one intravenous bolus of contrast material in approximately 30 seconds. ${ }^{11-13}$ SVCT allows continuous CT scanning of organ volumes during a single breath hold by advancing the patient through the $x$ ray beam.

\footnotetext{
5 June 1995

Revised version received

1 September 1995

Accepted for publication

4 September 1995

Department of
} 


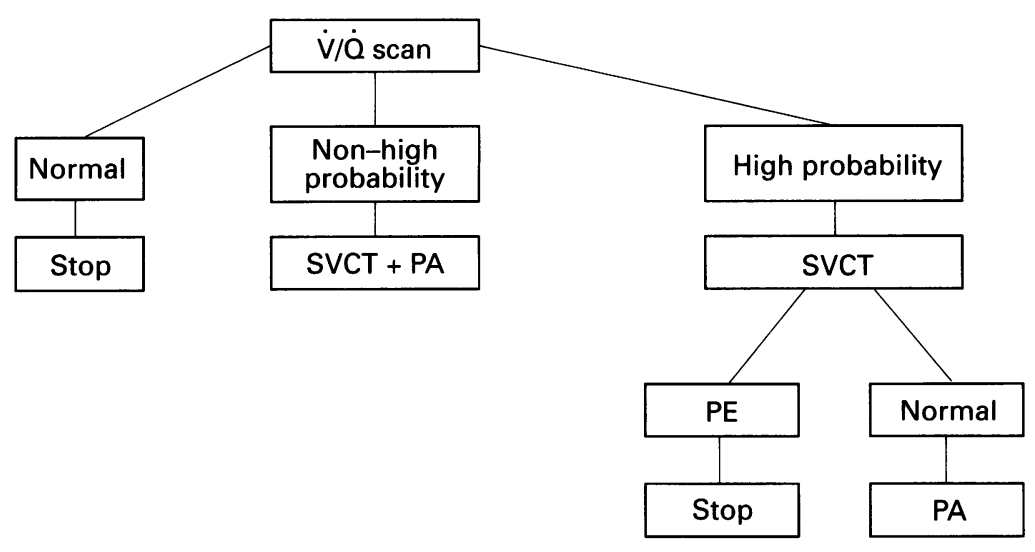

Figure 1 Flow chart of the study design. SVCT= spiral volumetric computed tomographic scanning; $P A=$ pulmonary angiography; $P E=$ pulmonary embolism.

The purpose of this prospective study was to confirm the potential role of SVCT in the detection of pulmonary emboli, and to define its value in the diagnostic strategy for pulmonary embolism.

\begin{abstract}
Methods
PATIENTS

From August 1993 to January 1995 a total of 348 consecutive patients had a $\mathbf{V} / \dot{Q}$ scan for clinical suspicion of pulmonary embolism based on history, physical examination, laboratory findings, and chest radiographic or electrocardiographic abnormalities, and were eligible for inclusion in the study. Exclusion criteria were the use of anticoagulants for more than 48 hours, contraindications for pulmonary angiography - for example, pulmonary hypertension or severe cardiac dysfunction as indicated by the referring physician - and contraindications to the use of intravenous contrast material such as hypersensitivity, severe congestive heart failure, and pregnancy. Fifty two patients were using anticoagulants for more than 48 hours, 25 patients refused to enter the study, 20 patients had severe cardiac dysfunction, one had a history of a severe allergic reaction to intravenous contrast medium, and one proved to have an aortic dissection at SVCT and subsequently went for thoracic surgery, so no pulmonary angiography could be performed. Thus, 249 patients entered the study after informed consent was obtained.
\end{abstract}

\section{DIAGNOSTIC STRATEGY}

If the $\dot{V} / \dot{Q}$ scan was normal, investigations for emboli were stopped and the patients were recorded not to have emboli. Patients with an abnormal $\dot{V} / Q$ Q scan underwent SVCT. If patients had a high probability $\dot{V} / \mathbf{Q}$ scan, we

Table 1 Spiral volumetric $C T$ findings versus $\dot{V} / \dot{Q}$ scan findings

\begin{tabular}{lllll}
\hline$\dot{V} / Q$ scan & \multicolumn{3}{l}{ Spiral volumetric $C T$} & \\
\cline { 2 - 5 } & $\begin{array}{l}\text { No pulmonary } \\
\text { embolism }\end{array}$ & $\begin{array}{l}\text { Pulmonary } \\
\text { embolism }\end{array}$ & Inconclusive & Total \\
\hline Non-high probability & 35 & 6 & 1 & 42 \\
High probability & 2 & 32 & 1 & 35 \\
Total & 37 & 38 & 2 & 77 \\
\hline
\end{tabular}

$\mathrm{CT}=$ computed tomography; $\dot{\mathrm{V}} / \dot{\mathrm{Q}}=$ ventilation/perfusion did not perform a pulmonary angiogram if the SVCT was also positive for pulmonary embolism in view of the very high reported positive predicted value of a high probability scan. ${ }^{6-9}$ Pulmonary angiography was only performed to establish a final diagnosis in those patients who had a high probability scan but whose SVCT scan was negative. If the $\dot{V} / \dot{Q}$ scan was neither normal nor high probability it was classified as non-diagnostic ${ }^{8101415}$ and in these patients we performed both SVCT and pulmonary angiography. These two examinations were always performed within 24 hours of each other (fig 1).

In addition, ultrasonography of the lower limb was performed on the same day to detect deep vein thrombosis in all patients with an abnormal perfusion scintigram.

\section{TECHNIQUES}

$\dot{V} / \dot{Q}$ scan

Technetium-99m labelled macroaggregated albumin $(0.03 \mathrm{mCi} / \mathrm{kg})$ was used for perfusion lung scanning. Six views were obtained: anterior, posterior, right and left lateral, and right and left posterior oblique. Ventilation was simultaneously performed with krypton- $81 \mathrm{~m}$ $(40 \mu \mathrm{Gy})$. These six views were obtained in 99 patients but in 40 only four views could be obtained. The quality of the $\dot{V} / \dot{Q}$ scans was determined by the condition of the patients. Some had ventilation difficulties, others were bedridden and therefore were hard to position correctly. $\dot{V} / \dot{Q}$ scans were classified according to a clinical classification suggested by Moser and Hull. ${ }^{810} 1415$

$\dot{\mathrm{V}} / \mathbf{Q}$ scans were reported as normal when no perfusion defects were present, as high probability when one or more large areas of mismatch were present, areas with no perfusion and normal ventilation. All other scans were classified as non-diagnostic - that is, they were not conclusive. $\dot{V} / \dot{Q}$ scans were interpreted together with chest radiographic appearances.

\section{$C T$ scan}

SVCT scans were performed using a Somatom Plus S scanner (Siemens, Erlangen, Germany). If possible, scanning was performed during a single breath hold, but inability to hold respiration was not considered a criterion for exclusion. If patients were short of breath they were asked to breathe gently. SVCT scans were obtained using $210 \mathrm{~mA}$ and $120 \mathrm{kV}$. A $360^{\circ}$ linear interpolation was used for the reconstruction of a single image. Scanning time was 32 seconds with a $5 \mathrm{~mm} / \mathrm{s}$ table feed resulting in a scanning volume of $16 \mathrm{~cm}$. Five $\mathrm{mm}$ slices were reconstructed with a $4 \mathrm{~mm}$ increment. In one patient the scan was inconclusive and an additional target scan with $2 \mathrm{~mm}$ slices of a specific area was made. As the scanning volume was restricted to $16 \mathrm{~cm}$, the SVCT range started at the top of the aortic arch so that the scan would cover all segmental pulmonary arteries. One hundred $\mathrm{ml}$ of a low osmolarity $30 \%$ iodinated non-ionic contrast agent (Iopamiro 300, Bracco, Italy) was administered via an antecubital vein using an 
A

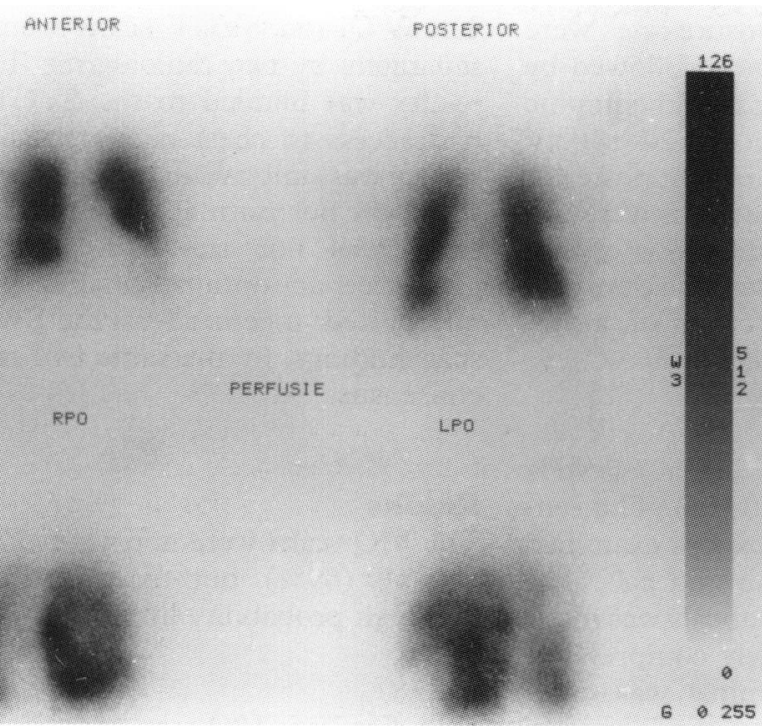

B
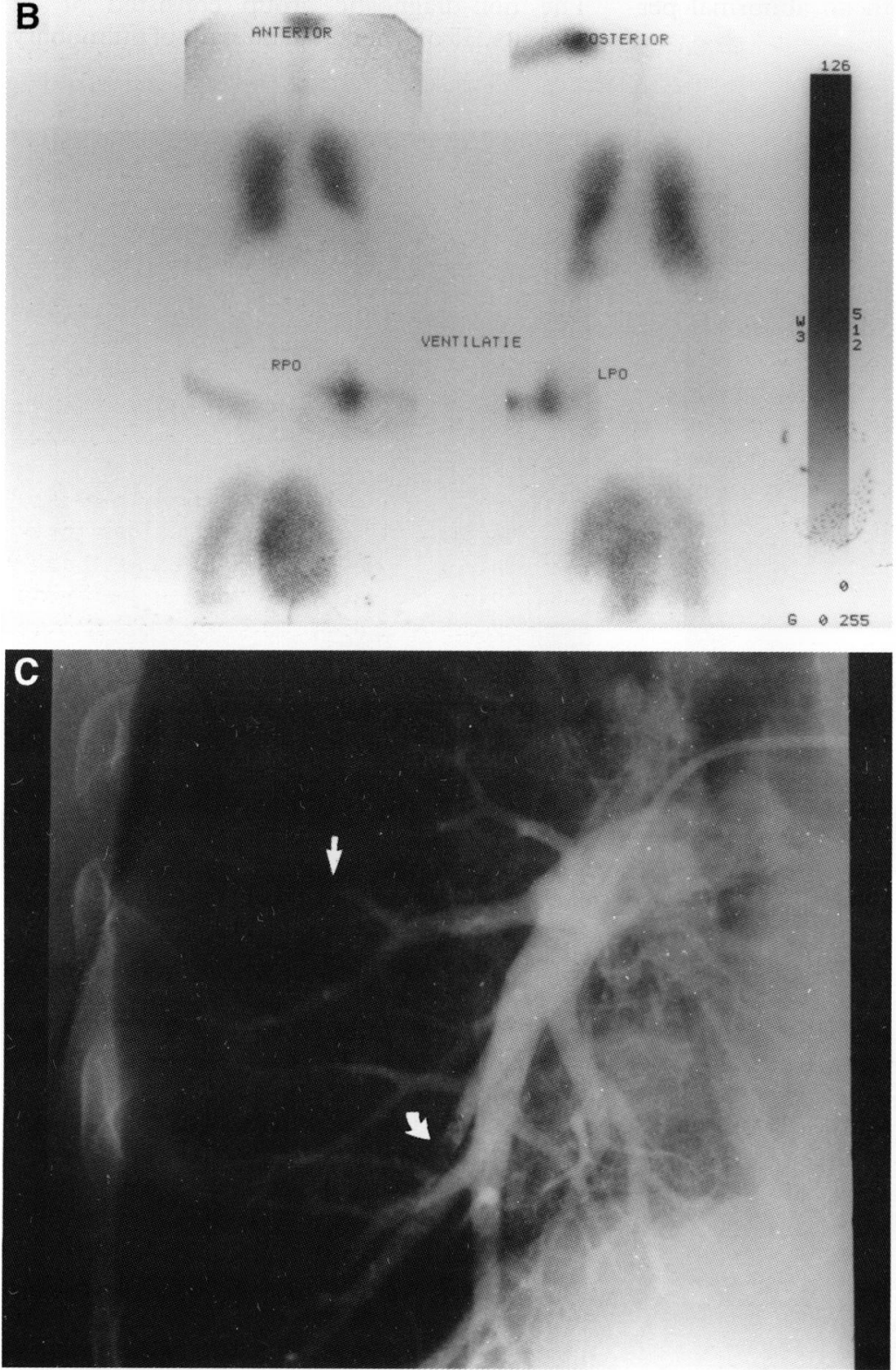

Figure 2 An 84 year old woman with sudden onset of dyspnoea and chest pain. $(A)$ and (B) The $\dot{V} / Q$ scan showing matching defects in the right posterobasal and lateral middle lobe segment, and in the left anterobasal segment. (C) The pulmonary angiogram of the right lung showing intraluminal filling defects in the anterobasal segmental artery (curved arrow) and in the lateral segmental artery (straight arrow), which could not be seen on SVCT scanning. automatic injector. We used a biphasic contrast injection with a flow of $3 \mathrm{ml} / \mathrm{s}$ during the first 15 seconds, then a flow of $2 \mathrm{ml} / \mathrm{s}$ up to $100 \mathrm{ml}$, with a 15 second scan delay. The entire examination required $10-15$ minutes. All images were viewed using lung (window width $1500 \mathrm{HU}$; window centre - $500 \mathrm{HU}$ ) and mediastinal (window width $400 \mathrm{HU}$; window centre $40 \mathrm{HU}$ ) settings.

The opacification of the pulmonary arteries was analysed using mediastinal window settings. Opacification was graded as good when it was adequate for detecting filling defects but the density was less than $200 \mathrm{HU}$, and as excellent when it demonstrated a high degree of vascular opacification $(>200 \mathrm{HU})$. SVCT scans were classified as negative or positive for pulmonary embolism. The diagnosis of pulmonary embolism was based solely on the presence of one or more filling defects in the pulmonary arteries. Indirect signs such as a wedge-shaped pleurally-based parenchymal shadow corresponding to a pulmonary infarct were best evaluated at lung window settings and were only used as a support of the diagnosis of pulmonary embolism. Ancillary findings such as pneumonia were scored separately.

With our SVCT protocol, pulmonary arteries had a good enhancement with an average of $200 \mathrm{HU}$ in the main pulmonary artery. The vascular opacification was excellent in 51 patients $(>200 \mathrm{HU})$ and good in 27 patients $(<200 \mathrm{HU})$. The quality of the images was reduced in one obese patient, in two patients who were unable to raise their arms above their head, and in 11 patients who were unable to hold their breath. In all but two patients the overall quality of the SVCT images was judged to be adequate for interpretation $(97 \%)$.

\section{Angiography}

Using the Seldinger technique a 5 French Grollman catheter (Cordis, Roden, The Netherlands) was inserted into the femoral vein. Pulmonary arterial pressure was measured in the pulmonary trunk, as a pulmonary arterial pressure of $>50 \mathrm{~mm} \mathrm{Hg}$ was a contraindication for continuation of the procedure.

Angiography was performed under ECG monitoring. A low osmolarity $30 \%$ iodinated non-ionic contrast agent (Iopamiro 300) was

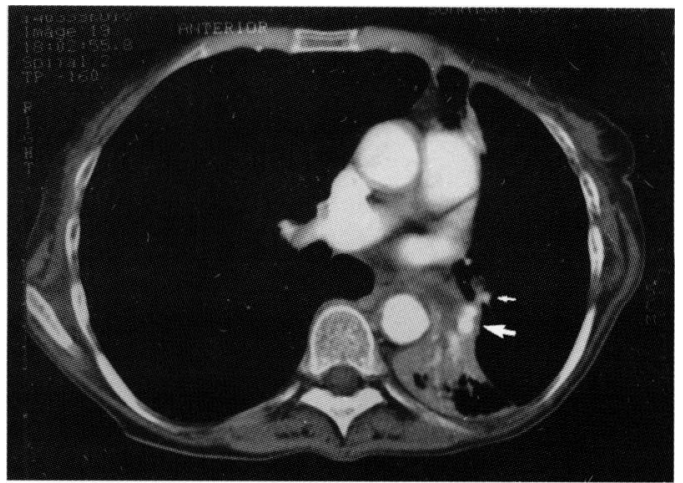

Figure 3 A 24 year old woman with a non-diagnostic $V / Q$ scan which showed a matching defect in the right lower lobe. SVCT scan showing normal enhancing right lower lobe arteries (white arrows). 
injected at a rate of $20-30 \mathrm{ml} / \mathrm{s}$ up to a total of $40-60 \mathrm{ml}$ in two seconds. Exposure rates were four per second for four seconds, followed by one per second for 4-6 seconds. Radiographic parameters were in the range of $120-130 \mathrm{kV}$ and 1.5-3 mA. Anteroposterior, right posterior and left posterior oblique projections were obtained. The criterion for the diagnosis of pulmonary embolism was the identification of a filling defect in, or an abrupt cutoff of, a pulmonary artery.

\section{Ultrasound}

Ultrasound was performed using a $7 \cdot 5 \mathrm{MHz}$ linear transducer (Toshiba $270 \mathrm{HE}$ ). The venous system of both lower limbs was examined from the inguinal canal to the mid calf. The diagnosis of deep venous thrombosis was made when the vein was incompletely compressible and/or an intraluminal thrombus was demonstrated. Ultrasonography was performed in 75 of the 77 patients with an abnormal perfusion lung scan.

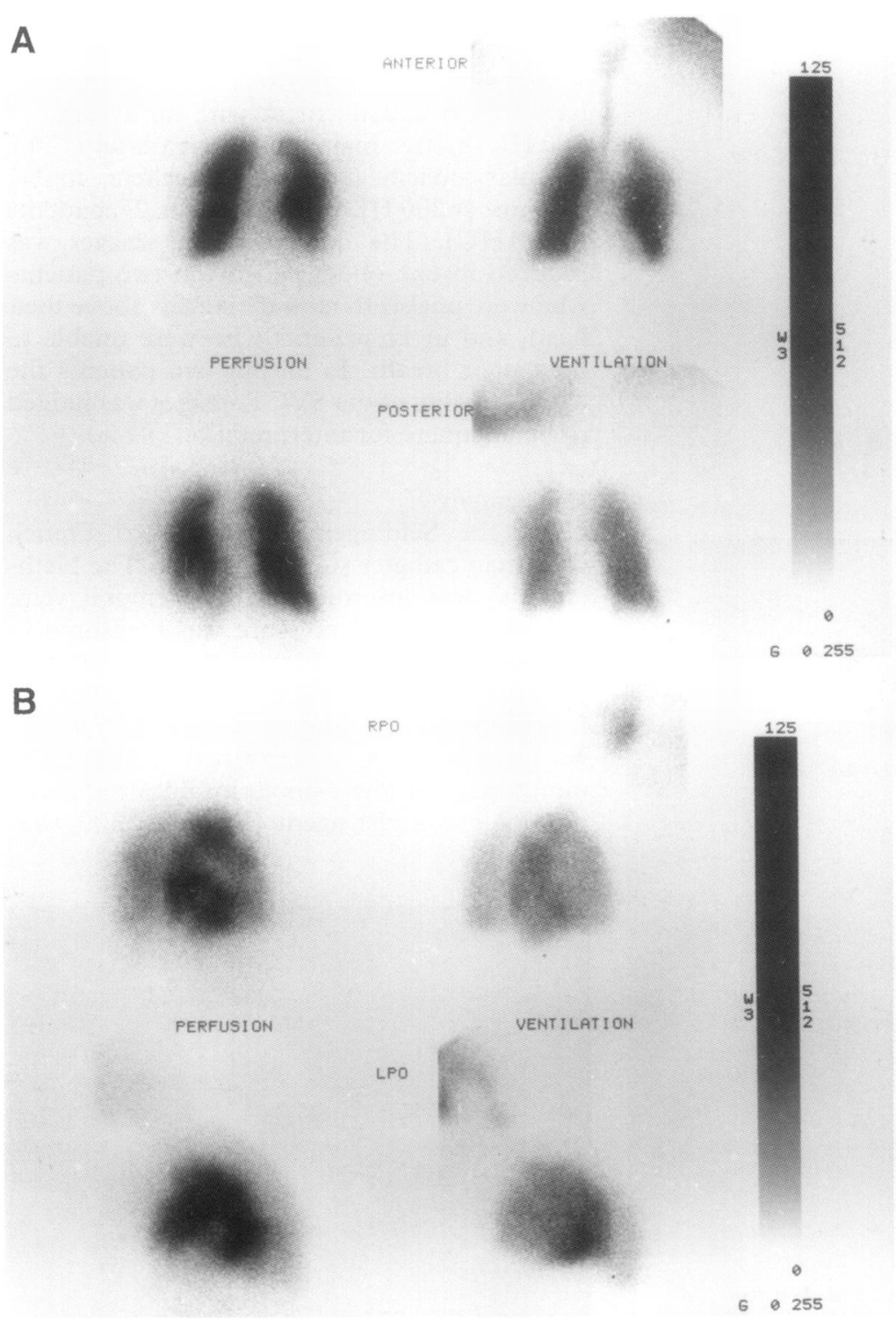

ASSESSMENT OF SCANS

The $\dot{V} / \dot{Q}$ and SVCT scans were interpreted separately by two radiologists. The $\dot{\mathrm{V}} / \mathrm{Q}$ scan reader was blinded to the SVCT results but had access to chest radiographs. The SVCT reader was only aware of the fact that the V/Q scan was not normal. The pulmonary angiogram was our standard of reference. To guarantee an optimal final diagnosis it was interpreted, together with the SVCT and $\dot{V} / \dot{Q}$ scan findings, by the same two radiologists in consensus.

\section{Results}

The $\dot{V} / \mathrm{Q}$ scans were normal in 172 of the 249 patients $(69 \%)$, non-diagnostic in $42(17 \%)$, and high probability in 35 (14\%) (table 1$)$.

Non-diagnostic $\dot{V} / \dot{Q}$ scan and SVCT findings The non-diagnostic group consisted of 42 patients, 35 of whom had no signs of pulmonary
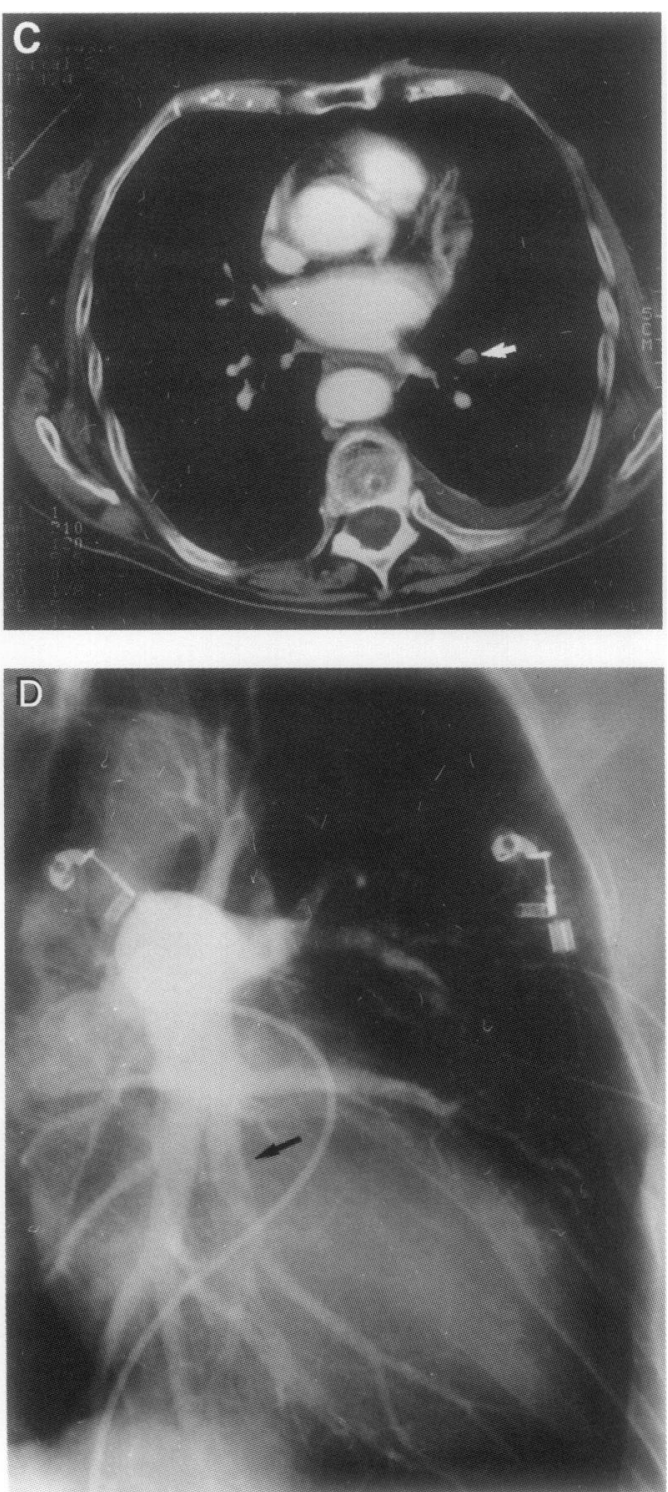

Figure $4 A 45$ year old woman with right sided chest pain in whom the $\dot{V} \mid \dot{Q}$ scan was non-diagnostic. (A) and (B) There is a partial mismatch on the posterior view in the basal segments of the left lung. LPO view shows a more matching defect in the basal segments. On the right are several peripheral small partly matching defects. (C) SVCT scan showing an intraluminal filling defect in the anterobasal segmental artery of the left lung (white arrow) and some pleural effusion (open arrow). (D) Pulmonary angiogram which confirmed the SVCT findings (black arrow). 

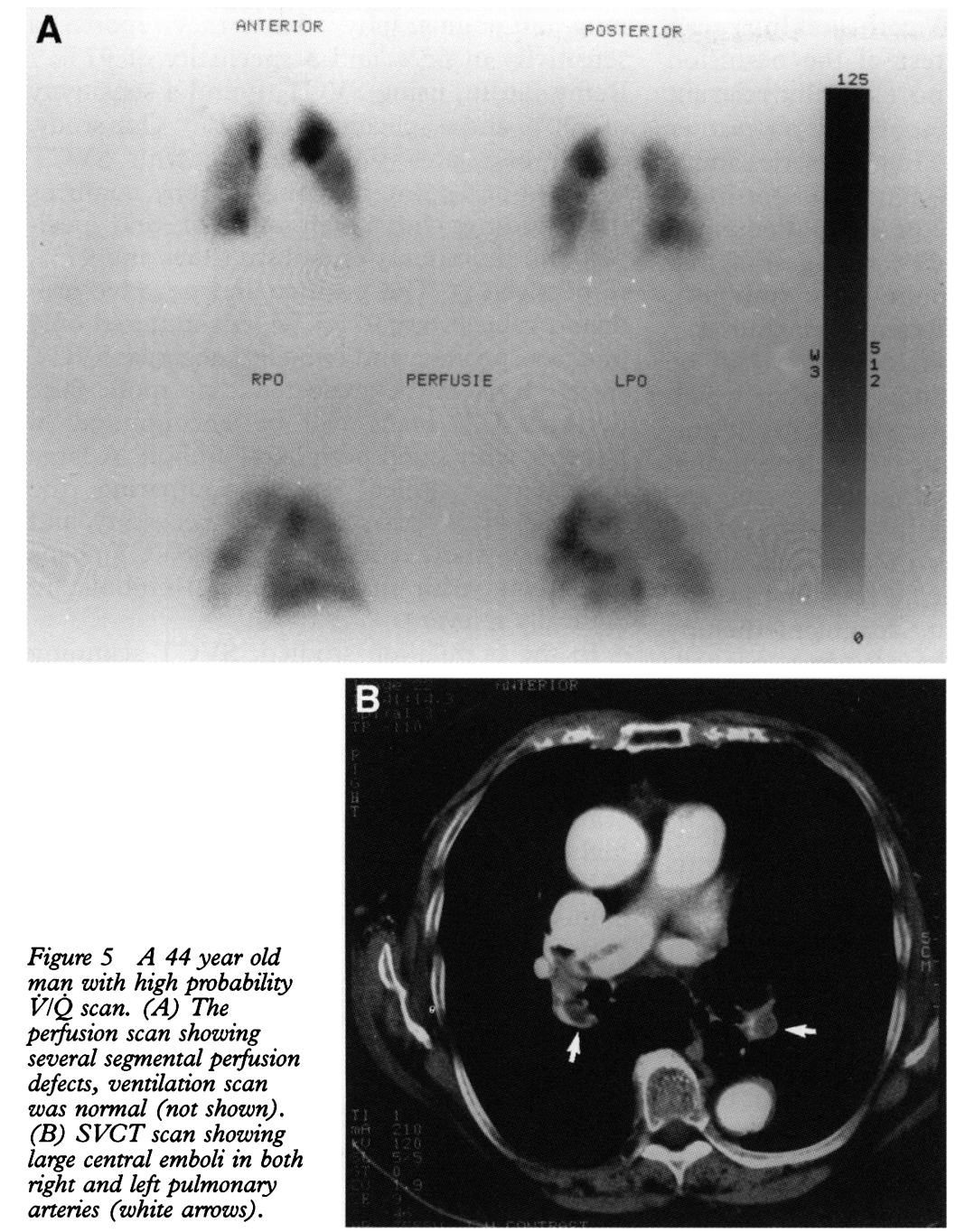

embolism on SVCT. In 34 of these 35 patients pulmonary angiography confirmed the SVCT findings with normal enhancing pulmonary arteries. One patient with a negative SVCT scan had a positive angiogram (fig 2).

Twenty four patients with a negative SVCT scan for pulmonary embolism showed other abnormalities - ranging from emphysema (6), pneumonia (9) (fig 3), pleural effusion (5), empyema (1), lung fibrosis (1), lymphadenopathy (1) to diaphragmatic hernia (1) which could explain the V/Q scan defects.

One of the 42 non-diagnostic patients had an inconclusive SVCT scan which was difficult to interpret because of moderate vascular opacification. There was no clear depiction of the right lower lobe arteries, making it impossible to rule out thromboembolism in this area. However, there was consolidation at the same location as the matching defects on the $\dot{V} / \dot{Q}$ scan. The pulmonary angiogram showed no pulmonary embolism. Six SVCT scans from the non-diagnostic group showed pulmonary emboli. In all but one case the diagnosis was confirmed by pulmonary angiography (fig 4). In one patient the angiogram was negative for pulmonary embolism (table 2).

Thus, six of the 42 patients with a nondiagnostic $\dot{\mathrm{V}} / \dot{\mathrm{Q}}$ scan proved to have pulmonary embolism and the SVCT scan was falsely negative in one of these.

High probability $\dot{V} / \dot{Q}$ scan and SVCT findings The group of high probability $\mathrm{V} / \mathrm{Q}$ scans consisted of 35 patients (14\%). In 32 cases the SVCT scans showed thromboembolism (fig 5). As for indirect signs of pulmonary embolism, consolidation interpreted as "pulmonary infarction" was seen in 10 cases and pleural effusion in eight. Several patients also showed other abnormalities such as emphysema (2) and two patients had a bronchial carcinoma. In two of the 35 patients with a high probability $\dot{V} / Q$ scan the SVCT scan showed no pulmonary emboli. In one of these the SVCT scan showed emphysema in the non-perfused areas, and in the other it was normal. Pulmonary angiography was negative in both cases. Finally, one of the 35 patients with a high probability $\dot{V} / \dot{Q}$ scan had an inconclusive SVCT scan and the pulmonary angiogram was positive.

In our population sensitivity and specificity of SVCT for pulmonary embolism were $95 \%$ and $97 \%$, respectively. The positive and negative predicted value of SVCT were both $97 \%$. Due to the study design the sensitivity of the $\dot{\mathrm{V}} / \mathbf{Q}$ scan was $100 \%$ by definition, and specificity was $82 \%$. In patients with an abnormal perfusion scan the positive predictive value of SVCT was $97 \%$ and of $\dot{V} / \dot{Q}$ scanning was $52 \%$. In the group of patients with a non-diagnostic $\dot{V} / \mathbf{Q}$ scan the positive predicted values of SVCT and $\dot{V} / Q \dot{Q}$ scans were $83 \%$ and $17 \%$, respectively.

\section{Ultrasonography}

Ultrasonography revealed deep vein thrombosis in 12 patients, all of whom had a high probability $\dot{\mathrm{V}} / \dot{\mathrm{Q}}$ scan and pulmonary embolism on the SVCT scan. Thus, in this study 12 out of 39 patients with pulmonary embolism had deep vein thrombosis $(31 \%)$.

\section{Discussion}

If a patient is suspected of having a pulmonary embolism there are several strategies to follow. One possibility is to treat all patients with anticoagulant drugs but, as pulmonary emboli are only found in a minority of those suspected, many patients would receive unnecessary and potentially harmful treatment. ${ }^{23}$

It is therefore imperative to establish or exclude the presence of pulmonary embolism (or deep vein thrombosis) in each patient suspected

Table $2 \dot{V} / \dot{Q}$ scan findings compared with spiral volumetric computed tomography (SVCT) and pulmonary angiography (PA) $(n=45)$

\begin{tabular}{|c|c|c|c|c|c|c|}
\hline$\dot{V} / \dot{Q}$ scan & $S V C T-/ P A-$ & $S V C T+/ P A+$ & $S V C T-/ P A+$ & $S V C T+/ P A-$ & $S V C T ? / P A-$ & $S V C T ? / P A+$ \\
\hline $\begin{array}{l}\text { Non-high probability } \\
\text { High probability }\end{array}$ & $\begin{array}{r}34 \\
2\end{array}$ & $\begin{array}{l}5 \\
-\end{array}$ & 1 & $\begin{array}{l}1 \\
-\end{array}$ & 1 & $\overline{1}$ \\
\hline
\end{tabular}


of having the condition. A perfusion lung scan is a sensitive screening test; if the perfusion scan is normal there is no clinically relevant pulmonary embolism present and no further investigation is necessary. ${ }^{4}$ In most series about $30-40 \%$ of patients had a normal perfusion scan. ${ }^{679}$ In our series 172 of 249 patients had a normal perfusion scan (69\%), suggesting that our clinicians consider pulmonary embolism too frequently in their differential diagnoses.

An abnormal perfusion lung scan can be caused by several conditions, some of which mimic the clinical signs and symptoms of pulmonary embolism. If a patient has an abnormal perfusion lung scan, additional tests are needed. One possibility is to try to detect the thrombosis in the pelvis or legs and ultrasonography is a very specific test for this. ${ }^{15}$ If a thrombosis is found, anticoagulant therapy is warranted and no additional test is needed. In our study additional tests would have been unnecessary in 12 out of 77 patients $(16 \%)$. Other authors have reported a similar limited value for ultrasonography in the diagnostic assessment of patients suspected of having pulmonary embolism. ${ }^{1617}$ If ultrasonography is negative for deep vein thrombosis, the diagnostic strategy has to be directed to the lungs themselves.

The usual next step is a ventilation-perfusion lung scan. If this scan shows one or more large segmental mismatches, there is a greater than $90 \%$ chance of pulmonary embolism being present, but only $10-20 \%$ of the patients suspected of pulmonary embolism will have such a high probability scan. ${ }^{6-9}$ In our series 35 patients had a high probability scan, and 32 of these had a positive SVCT scan. We felt that it was unnecessary to perform a pulmonary angiogram in these patients, as not only is the $\dot{\mathrm{V}} / \dot{\mathrm{Q}}$ scan specific in such patients, but the first studies with SVCT scanning also showed high specificity. ${ }^{11-13}$ Two patients with a high probability $\dot{V} / \dot{Q}$ scan had a negative SVCT scan and the pulmonary angiogram also showed no emboli. Therefore, in our population SVCT scanning was a good alternative to the ventilation scan in the high probability group.

The main issue in the diagnosis of pulmonary embolism is how to manage patients with a non-diagnostic $\dot{\mathrm{V}} / \dot{\mathrm{Q}}$ scan. According to the literature, $40-60 \%$ of patients suspected of pulmonary embolism will have such a $\dot{V} / \dot{Q}$ scan, and the frequency of pulmonary embolism in these patients varies from $10 \%$ to $40 \% .^{26-810} \mathrm{It}$ is therefore necessary to identify or exclude the presence of blood clots in the pulmonary arteries of those subjects. To date, pulmonary angiography remains the standard test for depicting pulmonary emboli, but there is often reluctance to perform this invasive procedure.

Fast CT scanning with bolus intravenous contrast media is a new, less invasive technique for studying the pulmonary arteries. It provides a straightforward diagnostic criterion - namely, the presence of intraluminal filling defects. First studies with SVCT and electron beam CT scanning were promising. ${ }^{11-13}$ Teigen et al found $85 \%$ agreement between findings at CT scan- ning and angiography. ${ }^{12}$ Later they reported a sensitivity of $65 \%$ and a specificity of $97 \% .^{13}$ Remy-Jardin, using SVCT, found a sensitivity of $100 \%$ and a specificity of $96 \% .{ }^{11}$ Our study, which correlates $\dot{V} / \dot{Q}$ scanning with SVCT scanning and pulmonary angiography, confirms these findings with a high sensitivity and specificity for pulmonary embolism $(95 \%$ and $97 \%$, respectively ). The positive and negative predicted values were $97 \%$. We encountered only one false positive and two false negative SVCT scans. It is to be expected that more false negative CT scans will be encountered in patients with small peripheral emboli. A large multicentre clinical study comparing the different diagnostic strategies is necessary, and will show whether false negative SVCT scans in patients with small peripheral emboli are clinically relevant.

In the population studied, SVCT scanning could have replaced ventilation scanning in patients with an abnormal perfusion scan. The sensitivity of SVCT scanning for pulmonary embolism was almost as high as that of $\dot{\mathrm{V}} / \dot{\mathrm{Q}}$ scanning (95\% versus $100 \%)$, specificity was higher $(97 \%$ versus $80 \%)$. The main benefit of SVCT scanning compared with $\dot{V} / \dot{Q}$ scanning is the high positive predicted value in patients with a non-diagnostic V/Q scan. SVCT scanning could also replace pulmonary angiography as the diagnostic test in patients with nondiagnostic $\dot{V} / \dot{Q}$ scans.

1 Dalen JE, Alpert JS. Natural history of pulmonary embolism. Prog Cardiovasc Dis 1975;259-70.

2 Robin ED. Overdiagnosis and overtreatment of pulmonary embolism: the emperor may have no clothes. Ann Intern Med 1977;87:775-81.

3 Levine MN, Raskob GE, Hirsh J. Hemorrhagic complications of long-term anticoagulant therapy. Chest 1989; 95:s26-35.

4 Hull RD, Raskob G, Hirsh J. Clinical validity of a normal perfusion lung scan in patients with suspected pulmonary embolism. Chest 1990;97:23-6.

5 Kipper MS, Moser KM, Kortman KE, Ashburn WL. Long term follow up of patients with suspected pulmonary embolism and a normal lung scan. Chest 1982;82:411-5.

6 PIOPED investigators. Value of the ventilation/perfusion scan in acute pulmonary embolism: results of the prospective investigation of pulmonary embolism diagnosis. fAMA 1990;263:2753-9.

7 Biello DR, Mattar A, McKnigt RC, Siegel BA. Ventilationperfusion studies in suspected pulmonary embolism. $A \mathscr{F} R$ 1979;133:1033-7.

8 Hull RD, Hirsh J, Carter CJ, Raskob GE, Gill GJ, Jay RM, et al. Diagnostic value of ventilation-perfusion scanning in patients with suspected pulmonary embolism. Chest

9 Hull RD, Raskob GE, Carter CJ, et al. Pulmonary embolism in outpatients with pleuritic chest pain. Arch Intern Med 1988;148:838-44.

10 Hull RD, Hirsh J, Carter C, Jay RM, Dodd PE, Ockkford $\mathrm{PA}$, et al. Pulmonary angiography, ventilation lung scanning and venography for clinical suspected pulmonary embolism with abnormal perfusion scan. Ann Intern Med 1983;98:891-9.

11 Remy-Jardin M, Remy J, Wattine L, Giraud F. Central pulmonary thromboembolism diagnosis with spiral voparison with pulmonary angiography. Radiology 1992;185 381-7.

12 Teigen CL, Maus TP, Sheedy PF, Johnson CM, Stanson AW, Welch TJ. Pulmonary embolism: diagnosis with electron beam CT. Radiology 1993;188:839-45.

13 Teigen CL, Maus TP, Sheedy PF, Stanson AW, Johnson $\mathrm{CM}$, Breen JF, et al. Pulmonary embolism: diagnosis with CM, Breen JF, et al. Pulmonary embolism: diagnosis with contrast enhanced electron-beam CT and comparison
with pulmonary angiography. Radiology 1995;194:313-9. 14 Hull RD, Raskob GE. Low probability lung scan findings a need for change. Ann Intern Med 1991;114:142-3. a need for change. Ann Intern Med 1991;114:142-3. 1990;141:235-49.

16 Cronan J. Venous thromboembolic disease: the role of US. Radiology 1993;186:619-30.

17 Quinn RJ, Nour R, Butler P, Glenn DW, Travers PL, Wellings G, et al. Pulmonary embolism in patients with intermediate probability lung scans: diagnosis with Doppler venous US and d-dimer measurement. Radiology 1994;190:509-11. 